\title{
Brain-derived neurotrophic factor in depression: a male problem?
}

\author{
Molecular Psychiatry (2010) 15, 227; \\ doi:10.1038/mp.2010.19
}

There has been considerable interest in the potential role of brain-derived neurotrophic factor (BDNF) in psychiatric disorders, including schizophrenia, bipolar disorder and major depression. BDNF is a member of the nerve growth factor superfamily, the importance of which was recognized by the Nobel Prize for Physiology or Medicine awarded to Stanley Cohen and Rita Levi-Montalcini in 1986. BDNF has key functions in the brain, including neuronal differentiation, synaptic plasticity, dendritic arborization and modulation of the establishment of neuronal circuits that regulate complex behavior. ${ }^{1}$ The $B D N F$ gene, localized on the short arm of chromosome 11 (11p13), has at least eight distinct promoters. Through alternative promoters, splicing and polyadenylation sites, over 18 transcripts can be generated, but amazingly they all encode an identical initial BDNF peptide. This complex transcriptional organization may provide multiple opportunities for regulation. ${ }^{1}$

Given the multi-layered regulation of the BDNF transcription process, there is a very long pathway from single-nucleotide polymorphisms (SNPs) of the $B D N F$ gene to changes in BDNF function that might underlie psychiatric symptoms. The hypothesis that a SNP in BDNF would affect BDNF function and cause psychiatric symptoms would require testing at various levels, from genetics to transcription regulation, gene expression, translation and function. Counterintuitively, the Val66Met SNP in $B D N F$, found only in humans, leading to a Met substitution for Val at codon 66 in the prodomain, may be a marker for BDNF function. This is a common polymorphism with an allele frequency of $20-30 \%$ in Caucasians and it correlates with reproducible phenotypes in human carriers. For example, heterozygosity for the Met allele is associated with smaller hippocampal volumes and several phenotypes. ${ }^{2}$ In Molecular Psychiatry alone we have published 165 papers on BDNF, showing myriad associations with the Val66Met SNP, from increased BDNF serum concentrations ${ }^{3}$ to normal hippocampal aging, as reported in last month's issue, ${ }^{4}$ and human memory and hippocampal function. ${ }^{5}$ Interestingly, Pezawas et al. ${ }^{6}$ recently showed evidence of biological epistasis between BDNF and the serotonin transporter (SLC6A4), with implications for depression, and Gatt et $a .^{7}$ showed that interactions between the BDNF Val66Met SNP and early-life stress predict brain and arousal pathways to syndromal depression and anxiety.

In the light of the associations between the BDNF Val66Met SNP and brain function, it is natural that a very large number of studies have emerged reporting associations between this SNP and major depression. As is typically the case, results from various studies are not concordant. A careful meta-analysis of existing studies is therefore required to determine whether there is indeed an association across populations. Such a study appears in this issue of Molecular Psychiatry. ${ }^{8}$ Verhangen et al. included in their meta-analysis 14 studies with a total of 2812 cases with DSM-III- or DSM-IV-defined major depressive disorder and 10843 nondepressed controls, including a study from our group. ${ }^{9}$ In the total sample, the BDNF Val66Met polymorphism was not significantly associated with depression. However, the gender-stratified analyses revealed significant effects in both the allelic and genotypic analyses in men but not in women. This provides further evidence for a potential basis for the sex ratio differences observed in the prevalence of major depression, which has a female to male ratio ranging from 2:1 to 5:2.

Of relevance to these findings are the observations of considerable genetic variation in BDNF allele frequencies across populations. Petryshen et al. ${ }^{10}$ carried out population genetic analyses of the BDNF genomic region and detected substantial variation in $B D N F$ coding region SNP allele and haplotype frequencies between 58 global populations, with the derived Met allele of Val66Met ranging in frequency from 0 to $72 \%$ across populations. In our own studies, we showed through deep sequencing of eight candidate genes, including $B D N F$, that in a Mexican-American population from Los Angeles, over $50 \%$ of the observed genetic variation was novel and was not described in public databases. ${ }^{11,12}$ Such novel variants were mostly of rare frequency. Given the key role of BDNF in brain function and the association with depression (at least in men), we suggest that a fruitful avenue for future research might be to directly re-sequence this gene in subjects with and without depression across various populations, to determine whether the impact of genetic variants of BDNF is a case of common disease-common variant (such as Val66Met) or of common disease-rare variant.

J Licinio and M-L Wong John Curtin School of Medical Research, The Australian National University, Canberra, Australian Capital Territory, Australia E-mail: julio.licinio@anu.edu.au

\section{References}

1 Greenberg ME et al. J Neurosci 2009; 29: 12764-12767.

2 Chen Z-Y et al. Science 2006; 314: 140-143.

3 Lang UE et al. Mol Psychiatry 2009; 14: 120-122.

4 Sambataro F et al. Mol Psychiatry 2010; 15: 116-118.

5 Egan MF et al. Cell 2003; 112: 257-269.

6 Pezawas L et al. Mol Psychiatry 2008; 13: 709-716.

7 Gatt JM et al. Mol Psychiatry 2009; 14: 681-695.

8 Verhagen $\mathrm{M}$ et al. Mol Psychiatry 2010; 15: 260-271.

9 Ribeiro L et al. Neuroreport 2007; 18: 1291-1293.

10 Petryshen TL et al. Mol Psychiatry. Advance online publication 3 March 2009; doi: 10.1038/mp.2009.24.

11 Licinio J et al. Arch Gen Psychiatry 2009; 66: 488-497.

12 Dong C et al. Mol Psychiatry 2009; 14: 1105-1118.
} 